\title{
BCS-BEC crossover in a random external potential
}

\author{
G. Orso $^{1}$ \\ ${ }^{1}$ Laboratoire Physique Théorique et Modèles Statistiques, \\ Université Paris Sud, Bat. 100, 91405 Orsay Cedex, France
}

\begin{abstract}
We investigate the ground state properties of a disordered superfluid Fermi gas across the BCSBEC (Bose Einstein condensate) crossover. We show that, for weak disorder, both the depletion of the condensate fraction of pairs and the normal fluid density exhibit a nonmonotonic behavior as a function of the interaction parameter $1 / k_{F} a$, reaching their minimum value near unitarity. We find that, moving away from the weak coupling BCS regime, Anderson's theorem ceases to apply and the superfluid order parameter is more and more affected by the random potential.
\end{abstract}

PACS numbers:

Ultracold Fermi gases near a Feshbach resonance have been the subject of intense experimental and theoretical investigations over the last five years [1]. By tuning the inverse s-wave scattering length $1 / a$ continuously from $-\infty$ to $+\infty$, the ground state of these systems transforms from a weak-coupling BCS superfluid into a BoseEinstein condensate of diatomic molecules.

An interesting and general problem is to explore the BCS-BEC crossover in the presence of a random external potential. Optical disorder can be introduced in ultracold gases in a highly controllable way. For instance, in recent experiments with elongated atomic BEC [2, 3, 4], speckle patterns, created by a laser beam passing through a diffusive plate, were shown to suppress the 1D expansion of the gas. A characteristic feature of these random potentials is that they are slowly varying in space, with a typical length scale of the order of a few $\mu \mathrm{m}$. In order to produce a real white noise disorder, which is ubiquitous in condensed matter theory, it was recently suggested [5] to (randomly) trap atoms of a different species at the nodes of a tight 3D optical lattice. If the optical polarizability of the fermions is small, the latter will not experience the periodic potential but only the interaction with the impurity atoms.

To date, the effects of impurities on a superfluid Fermi gas near unitarity $(1 / a=0)$ are unknown, even for weak disorder. For s-wave BCS superconductors, Anderson's theorem [6] states that the (disorder-averaged) order parameter is unaffected by nonmagnetic impurities, as long as localization effects can be neglected. The superfluid density, measuring the dynamical response of the system to a superfluid flow, is instead reduced by impurities [7]. For Bose superfluids, the depletions of the condensate fraction and of the superfluid density induced by a weak disorder have been calculated in Refs [8, 9].

In this Letter we provide the first theoretical study of a disordered superfluid Fermi gas across the BCS-BEC crossover, at zero temperature. We show that the depletion of the condensate fraction of pairs and the normal fluid density, induced by a weak disorder, exhibit nonmonotonic behavior as a function of the interaction strength, with a pronounced minimum near unitarity.
These results are therefore consistent with the general expectation that superfluidity is more robust against suppressing mechanisms in the crossover regime. Nonmonotonic behaviors across the resonance have recently been observed 10] in critical velocities for superfluid flow, in agreement with earlier theoretical predictions [1, 12].

Our work is based on the Nozieres and Schmitt-Rink (NSR) theory 13 of superconducting fluctuations, extended to the broken symmetry state [14]. In particular, we will make use of techniques developed in Ref.[15], where the NSR theory has been applied to calculate the condensate fraction and the normal fluid density of clean Fermi gases across the BCS-BEC crossover.

In the following we assume that the random potential $V(\mathbf{r})$ originates from the scattering of fermions against impurity atoms and is given by $V(\mathbf{r})=\sum_{j} g_{d} \delta\left(\mathbf{r}-\mathbf{R}_{j}\right)$, where $g_{d}$ is the fermion-impurity coupling constant and $\mathbf{R}_{j}$ are the static positions of the impurities. The corresponding correlation function takes the white noise form $\langle V(-q) V(q)\rangle=\beta \delta_{i \omega_{m}, 0} \kappa$, where $q=\left(\mathbf{q}, i \omega_{m}\right)$ and $\kappa=n_{i} g_{d}^{2}, n_{i}$ being the concentration of impurities.

The two-component Fermi gas is described by the imaginary time action $S=\int_{0}^{\beta} d \tau \int d \mathbf{r}\left\{\bar{\psi}_{\sigma} \partial_{\tau} \psi_{\sigma}+H\right\}$, where $\beta$ in the inverse temperature and

$$
H=\bar{\psi}_{\sigma}(K+V) \psi_{\sigma}-g \bar{\psi}_{\uparrow}^{\dagger} \bar{\psi}_{\downarrow}^{\dagger} \psi_{\downarrow} \psi_{\uparrow} .
$$

Here $K=-\nabla^{2} / 2 m-\mu, \mu$ is the chemical potential, $g$ is the fermion-fermion interaction and we set $\hbar=$ Volume $=$ $k_{B}=1$.

Following Ref. 15], we apply the Hubbard-Stratonovich transformation to the Hamiltonian (1), by introducing a new bosonic field $\Delta(x)$ which couples to $\psi_{\uparrow} \psi_{\downarrow}$. Integrating out the fermionic fields, the partition function of the system takes the path integral form $Z=$ $\int D[\Delta, \bar{\Delta}] e^{-S \text { eff }}$, where

$$
S_{\mathrm{eff}}=\int_{0}^{\beta} d \tau \int d \mathbf{r}\left\{\frac{|\Delta(x)|^{2}}{g}-\frac{1}{\beta} \operatorname{Tr} \ln \left[-\beta \mathbf{G}^{-1}(x)\right]\right\}
$$

is the effective action written in terms of the inverse single particle Green's function

$$
\mathbf{G}^{-1}(x)=-\partial_{\tau}-(K+V(\mathbf{r})) \tau_{3}+\Delta(x) \tau^{+}+\bar{\Delta}(x) \tau^{-} .
$$


Here $x=(\mathbf{r}, \tau), \tau_{i}$ are the Pauli matrices and $\tau^{ \pm}=$ $\left(\tau_{1} \pm i \tau_{2}\right) / 2$.

To procede further, we assume that the main contribution to the partition function comes from small fluctuations $\delta \Delta(x)=\Delta(x)-\Delta$ around the uniform BCS pairing field $\Delta$. Hence, we expand the effective action (2) up to quadratic order in the bosonic fields $\delta \Delta$ and in the random potential $V$. To this purpose, the Green's function (3) is conveniently written as $\mathbf{G}^{-1}=\mathbf{G}_{0}^{-1}+\boldsymbol{\Sigma}$, where $\mathbf{G}_{0}^{-1}=-\partial_{\tau}-K \tau_{3}+\Delta \tau_{1}$ is the inverse BCS Green's function in the absence of disorder, and $\boldsymbol{\Sigma}=$ $-V(\mathbf{r}) \tau_{3}+\delta \Delta(x) \tau^{+}+\overline{\delta \Delta}(x) \tau^{-}$.

Averaging over the positions of the impurity atoms, we obtain $S_{\text {eff }}=S_{F}+A(\delta \Delta(0)+\overline{\delta \Delta}(0))+S_{B}$, where $S_{F}=\beta \Delta^{2} / g-\beta^{-1} \sum_{k} \operatorname{Tr} \ln \left[-\mathbf{G}_{0}^{-1}(k)+V(0) \tau_{3}\right]+\beta \Omega_{F}^{d}$ corresponds to the mean field action. Here $\mathbf{G}_{0}^{-1}(k)=$ $i \omega_{n}-\xi_{\mathbf{k}} \tau_{3}+\Delta \tau_{1}$ is written in momentum representation, with $\xi_{\mathbf{k}}=|\mathbf{k}|^{2} / 2 m-\mu$ and

$$
\Omega_{F}^{d}=\frac{1}{2 \beta} \sum_{k q} \operatorname{Tr}\left[\mathbf{G}_{0} \tau_{3} \mathbf{G}_{0}^{\prime} \tau_{3}\right]\langle V(q) V(-q)\rangle,
$$

where $\mathbf{G}_{0}=\mathbf{G}_{0}(k)$ and $\mathbf{G}_{0}^{\prime}=\mathbf{G}_{0}(k+q)$. Furthermore, $A=\Delta\left(1 / g-\sum_{\mathbf{k}} \tanh \left(\beta E_{\mathbf{k}} / 2\right) / 2 E_{\mathbf{k}}\right)$, where $E_{\mathbf{k}}=$ $\left(\xi_{\mathbf{k}}^{2}+\Delta^{2}\right)^{1 / 2}$ is the energy spectrum of single particle excitations, and

$$
S_{B}=\frac{1}{2} \sum_{q}\left[\eta^{\dagger} \mathbf{M} \eta+V(-q) W^{\dagger} \eta+V(q) W \eta^{\dagger}\right]
$$

corresponds to the gaussian action for the bosonic fluctuations. Here $\eta^{\dagger}=[\delta \bar{\Delta}(q), \delta \Delta(-q)]$ and $\mathbf{M}$ is a $2 \times 2$ symmetric matrix whose elements are given by

$$
M_{11}(q)=\frac{1}{g}+\sum_{k} G_{0,22} G_{0,11}^{\prime}, \quad M_{12}(q)=\sum_{k} G_{0,12} G_{0,12}^{\prime},
$$

and $M_{22}(q)=M_{11}(-q)$ (explicit expressions can be found in Ref.[15]). In Eq.(15), the doublet

$$
W(q)=\left(\begin{array}{c}
\sum_{k} G_{0,12} G_{0,11}^{\prime}-G_{0,22} G_{0,12}^{\prime} \\
\sum_{k} G_{0,11} G_{0,12}^{\prime}-G_{0,12} G_{0,22}^{\prime}
\end{array}\right),
$$

couples disorder to the bosonic fluctuations and induces processes in which pairs are scattered in or out of the condensate. At zero temperature, $W_{1}=W_{2}=\sum_{\mathbf{k}} \Delta\left(\xi_{\mathbf{k}}+\right.$ $\left.\xi_{\mathbf{k}+\mathbf{q}}\right) / 2 E_{\mathbf{k}} E_{\mathbf{k}+\mathbf{q}}\left(E_{\mathbf{k}}+E_{\mathbf{k}+\mathbf{q}}\right)$, for $q=(\mathbf{q}, 0)$.

By assumption, the mean field action $S_{F}$ is an extremum of $S_{\text {eff }}$, hence the coefficient $A$ must be set equal to zero $(A=0)$. This yields the usual BCS gap equation $1 / g=\sum_{\mathbf{k}} \tanh \left(\beta E_{\mathbf{k}} / 2\right) / 2 E_{\mathbf{k}}$, as in the $a b$ sence of impurities. This equation is ultraviolet divergent and is cured [14] by replacing the bare coupling constant $g$ with the scattering length $a$ according to $1 / g=-m / 4 \pi a+\sum_{\mathbf{k}} 1 / 2 \epsilon_{\mathbf{k}}$, with $\epsilon_{\mathbf{k}}=\mathbf{k}^{2} / 2 m$. At zero temperature, one finds

$$
-\frac{m}{4 \pi a}=\sum_{\mathbf{k}}\left[\frac{1}{2 E_{\mathbf{k}}}-\frac{1}{2 \epsilon_{\mathbf{k}}}\right]
$$

which should be seen as a self-consistent relation between $\Delta$ and $\mu$.

In the NSR theory, the second constraint is provided by the number equation $n=-\partial \Omega / \partial \mu$, where $\Omega=-\ln Z / \beta$ is the thermodynamic potential. To calculate it, we substitute $S_{\text {eff }}=S_{F}+S_{B}$ in the partition function $Z$ and integrate over the Bose fields. At zero temperature, this gives $\Omega=\Omega_{F}+\Omega_{B}$, where $\Omega_{F}=S_{F} / \beta$ is the mean field fermionic term and

$$
\Omega_{B}=\lim _{\beta \rightarrow \infty} \frac{1}{2 \beta} \sum_{q} \ln \operatorname{det} M-\frac{\kappa}{2} \sum_{\mathbf{q}, \omega_{m}=0} W^{\dagger} \mathbf{M}^{-1} W
$$

gives the contribution from bosonic fluctuations.

The integration over $\mathbf{q}$ in Eq.(4) is also ultraviolet divergent, due to the zero range nature of the fermionimpurity potential. This problem is solved by a renormalization of the related coupling constant $g_{d} \rightarrow g_{d}+$ $g_{d}^{2} \sum_{\mathbf{q}} 1 / \epsilon_{\mathbf{q}}$. Equation (4) then yields $\Omega_{F}^{d}=\kappa \Delta^{2} m^{3} / 4 \pi^{2}$, which is independent of $\mu$. As a result, the number equation takes the form

$$
n=\sum_{\mathbf{k}}\left(1-\frac{\xi_{\mathbf{k}}}{E_{\mathbf{k}}}\right)-\frac{\partial \Omega_{B}}{\partial \mu} .
$$

Differently from Eq.(8), Eq.(10) depends explicitly on disorder through the contribution (91) from bosonic fluctuations. From Eqs (8) and (10) we obtain $\Delta$ and $\mu$ as a function of the atom density $n=k_{F}^{3} / 3 \pi^{2}$, the scattering length $a$ and the disorder strength $\kappa$ (up to linear terms).

In the BCS limit, corresponding to $1 / k_{F} a \rightarrow-\infty$, the bosonic contribution in Eq.(10) is negligible and we find $\mu=\epsilon_{F}=k_{F}^{2} / 2 m$ while Eq.(8) gives $\Delta=$ $8 e^{-2} \epsilon_{F} \exp \left(-\pi / 2 k_{F}|a|\right)$, as in the absence of disorder. This means that for weak attraction the superfluid order parameter is unaffected by the disorder, in agreement with Anderson's theorem [6].

In the opposite strong coupling regime, corresponding to $1 / k_{F} a \rightarrow+\infty$, the gap equation (8) yields $\mu=$ $-1 / 2 m a^{2}$, which is half of the binding energy. To find $\Delta$, we first differentiate Eq.(9) with respect to $\mu$ and then expand the obtained result in powers of $q$, assuming $|\mathbf{q}|^{2} / 2 m, i \omega_{m} \ll|\mu|$. Taking into account that $W_{1}(\mathbf{q}=0)=m^{2} a \Delta / 4 \pi$, from Eq. (10) we obtain

$$
\frac{n}{2}=\frac{m^{2} \Delta^{2} a}{8 \pi}+\sum_{\mathbf{q}}\left[\frac{\epsilon_{\mathbf{q}}^{M}+\mu_{M}}{2 \omega_{\mathbf{q}}}-\frac{1}{2}\right]+4 \kappa \sum_{\mathbf{q}} \frac{m^{2} \Delta^{2} a / 8 \pi}{\left(\epsilon_{\mathbf{q}}^{M}+2 \mu_{M}\right)^{2}},
$$

where $\epsilon_{\mathbf{q}}^{M}=\mathbf{q}^{2} / 4 m, \omega_{\mathbf{q}}=\sqrt{\epsilon_{\mathbf{q}}^{M}\left(\epsilon_{\mathbf{q}}^{M}+2 \mu_{M}\right)}$ is the spectrum of the bosonic excitations. In Eq.(11), $\mu_{M}=$ $\Delta^{2} a^{2} m / 2=2 \pi a n / m$ is the effective chemical potential for the composite bosons corresponding to a molecular scattering length $a_{M}=2 a$ [16].

Equation (11) has a direct physical meaning: the term $\left(m^{2} \Delta^{2} a / 8 \pi\right)$ corresponds to the fraction of condensed molecules whereas the second term yields the Bogoliubov 


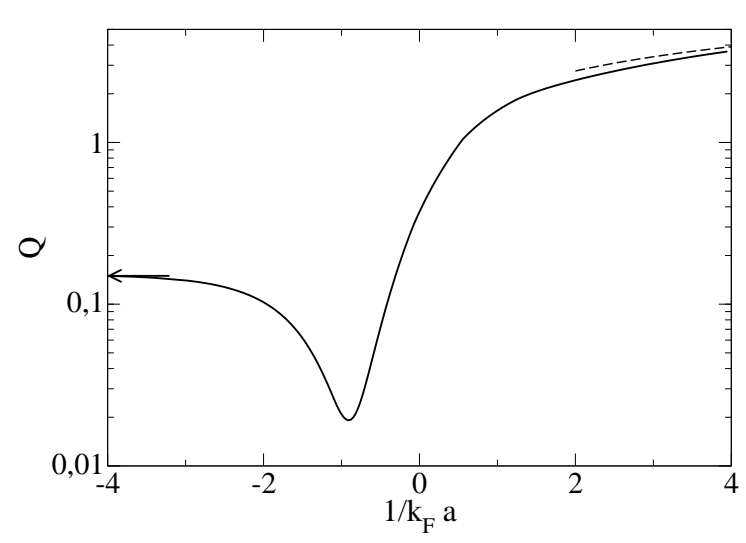

FIG. 1: Quantum depletion $\alpha-\alpha_{0}=-\left(\kappa m^{2} / k_{F}\right) Q\left(1 / k_{F} a\right)$ of the condensate fraction of pairs $\alpha$ induced by disorder: the function $Q\left(1 / k_{F} a\right)$ is shown with the solid line. The asymptotic behavior at strong coupling is shown with the dashed line. Notice that $Q$ saturates to $3 / 2 \pi^{2}$ at weak coupling (arrow).

quantum depletion due to the molecule-molecule interaction, as shown in Ref.[15]. The last term in Eq.(11) gives the density of molecules pushed out of the condensate by the random potential. In particular, the factor 4 accounts for the fact that the effective disorder potential $V_{M}$ seen by the molecule is $V_{M}(\mathbf{r})=2 V(\mathbf{r})$, hence the corresponding correlation function is given by $\left\langle V_{M}(\mathbf{q}) V_{M}(-\mathbf{q})\right\rangle=4 \kappa$.

From Eq.(11) we also find $\Delta-\Delta_{0}=-2 \kappa m / \pi a$, where $\Delta_{0}$ is the order parameter in the absence of disorder, showing that Anderson's theorem does not apply in the BEC limit. In particular, the correction $\Delta-\Delta_{0}$ diverges for $a \rightarrow 0^{+}$. This signals that in the absence of repulsive interactions, the uniform Bose gas of molecules is unstable against collapse into a localized orbital of the disorder potential [8].

Condensate fraction of pairs. This is defined as the dimensionless ratio $\alpha=2 n_{c} / n$, where $n_{c}=\int \mid G_{12}(\mathbf{r}, \tau=$ $0)\left.\right|^{2} d \mathbf{r}$ is the density of condensed pairs corresponding to the normalization of the Cooper pair wavefunction. In the absence of disorder, the condensate fraction is an increasing function of the interaction strength $1 / k_{F} a$. It has been calculated within the NSR approach in Ref. 15. and compared with previous mean field [17] and Quantum Monte Carlo [18] predictions.

We notice that disorder enters the formula $\mathbf{G}^{-1}=$ $\mathbf{G}_{0}^{-1}+\boldsymbol{\Sigma}$ in two different ways: i) directly, through the "self energy" $\boldsymbol{\Sigma}$, and ii) in the mean field propagator $\mathbf{G}_{0}$, through the shifts $\delta \mu=\mu-\mu_{0}, \delta \Delta=\Delta-\Delta_{0}$ of the variational parameters, as calculated from Eqs (8) and (10). Hence, the change in the density of condensed pairs is given by

$$
n_{c}-n_{c 0}=\frac{\partial n_{c}^{\mathrm{mf}}}{\partial \mu} \delta \mu+\frac{\partial n_{c}^{\mathrm{mf}}}{\partial \Delta} \delta \Delta+\sum_{\mathbf{k}} \frac{\Delta}{E_{\mathbf{k}}} \int \frac{d \omega}{2 \pi} \delta G_{12}^{d}
$$

where $n_{c}^{\mathrm{mf}}=\sum_{\mathbf{k}} \Delta^{2} / 4 E_{\mathbf{k}}^{2}$ is the mean field prediction for the condensate fraction and $\delta \mathbf{G}^{d}(k)$ corresponds to the disorder correction of the Green's function obtained by expanding the latter in powers of $\boldsymbol{\Sigma}$ and retaining up to second order terms.

We have calculated the rhs of Eq. (12) numerically. We find $\alpha-\alpha_{0}=-\left(\kappa m^{2} / k_{F}\right) Q\left(1 / k_{F} a\right)$, where the crossover function $Q\left(1 / k_{F} a\right)$ is plotted in Fig 1 with the solid line. We see that the depletion of the condensate fraction exhibits a pronounced minimum at $1 / k_{F} a \approx-0.9$.

In the deep BCS limit the leading contribution to the depletion (12) comes from $\delta G_{12}^{d}$. Neglecting bosonic fluctuations, we have $\delta \mathbf{G}^{d}(\mathbf{k}, i \omega)=\kappa \sum_{\mathbf{q}} \mathbf{G}_{0} \tau_{3} \mathbf{G}_{0}^{\prime} \tau_{3} \mathbf{G}_{0}$. From Eq. (12) we then find $n_{c}-n_{c 0}=-\kappa m^{2} k_{F}^{2} / 4 \pi^{4}$ corresponding to $Q=3 / 2 \pi^{2}$, as shown by the arrow in Fig 1. In the clean limit the condensate fraction $n_{c 0}=m k_{F} \Delta / 8 \pi$ vanishes exponentially as $1 / k_{F} a \rightarrow$ $-\infty$, so the effects of disorder are clearly important in this regime, despite the fact that the order parameter itself is not affected by impurities. This interesting result follows by noticing that, in the presence of disorder, the zeroth-order Green's function $G_{0,12}(\mathbf{r}, \tau=0)$ in coordinate space is damped by the factor $\exp (-r / 2 \ell)$, where $\ell=\pi / \kappa m^{2}$ is the mean free path between collisions [7]. Hence the order parameter $\Delta=g G_{12}(\mathbf{r}=0,0)$ is the same as in pure superfluids, while the condensate fraction $n_{c}=\int\left|G_{0,12}(\mathbf{r}, 0)\right|^{2} e^{-r / \ell} d \mathbf{r} \simeq \int\left|G_{0,12}(\mathbf{r}, 0)\right|^{2}(1-r / \ell) d \mathbf{r}$ is severely suppressed by disorder. Here the condition of weak disorder $\left(n_{c 0}-n_{c}\right) / n_{c 0} \ll 1$ yields $\kappa m k_{F} / \Delta \ll 1$.

In the BEC regime, the leading contribution to the depletion (12) comes from the disorder induced change $\delta \Delta$ of the order parameter and we recover $n_{c}-$ $n_{c 0}=-\kappa \Delta m^{3} / 2 \pi^{2}$, in agreement with Eq.(11) and Ref. [8]. The corresponding asymptotic behavior $Q=$ $(12 / \pi)^{1 / 2} / \sqrt{k_{F} a}$ is shown in Fig 1 with the dashed line. We see that the molecular gas is fully depleted if the scattering length vanishes. Notice however that weak disorder implies $\kappa m^{2} / k_{F}^{3 / 2} a^{1 / 2} \ll 1$, so one cannot take the limit $a \rightarrow 0^{+}$for fixed disorder $\kappa$.

Normal fluid density. This is defined as $\rho_{n}=n-\rho_{s}$, where $\rho_{s}$ is the superfluid density. The latter is related to the lowest order change $\Omega\left[Q_{z}\right]-\Omega[0]=\rho_{s} m v_{s}^{2} / 2$ in the thermodynamic potential of the gas in the presence of a supercurrent with velocity $v_{s}=Q_{z} / 2 m$ along, say, the z-axis. At zero temperature and in the absence of disorder, the superfluid density coincides with the total density. Therefore $\rho_{n}=-\lim _{Q_{z} \rightarrow 0} 4 m \partial^{2} \Omega^{d}\left[Q_{z}\right] / \partial Q_{z}^{2}$, where $\Omega^{d}\left[Q_{z}\right]$ is the contribution to the thermodynamic potential coming from disorder.

To apply this formula, we notice that the currentcarrying state is related to the equilibrium state by the gauge transformation $\Delta(\mathbf{r}) \rightarrow \Delta(\mathbf{r}) e^{i Q_{z} z}$. Accordingly, the BCS Green's function $\mathbf{G}_{0}$ maps into $\widetilde{\mathbf{G}}_{0}$, where [15]

$$
\widetilde{\mathbf{G}}_{0}^{-1}(k)=\left(i \omega_{n}-\frac{k_{z} Q_{z}}{2 m}\right)-\left(\xi_{\mathbf{k}}+\frac{Q_{z}^{2}}{8 m}\right) \tau_{3}+\Delta \tau_{1} .
$$




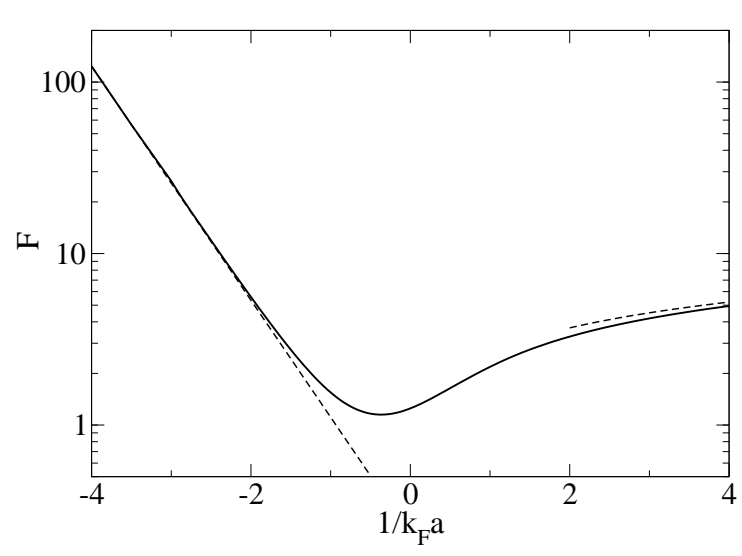

FIG. 2: Zero temperature normal density $\rho_{n} / n=$ $\left(\kappa m^{2} / k_{F}\right) F\left(1 / k_{F} a\right)$ induced by disorder: the function $F\left(1 / k_{F} a\right)$ is plotted with the solid line. The asymptotic behaviors at weak and strong coupling are shown with dashed lines.

Repeating the calculations from Eq.(4) to Eq.(9) with $\mathbf{G}_{0}$ replaced by $\widetilde{\mathbf{G}}_{0}$, we obtain

$$
\Omega^{d}\left[Q_{z}\right]=\frac{\kappa}{2} \sum_{q, k} \operatorname{Tr}\left[\widetilde{\mathbf{G}}_{0} \tau_{3} \widetilde{\mathbf{G}}_{0}^{\prime} \tau_{3}\right]-\frac{\kappa}{2} \sum_{q} \widetilde{W}^{\dagger} \widetilde{\mathbf{M}}^{-1} \widetilde{W}
$$

where $\widetilde{\mathbf{G}}_{0} \equiv \widetilde{\mathbf{G}}_{0}(k)$ and $\widetilde{\mathbf{G}}_{0}^{\prime} \equiv \widetilde{\mathbf{G}}_{0}(k+q)$, with $q=(\mathbf{q}, 0)$. In Eq.(14), $\widetilde{\mathbf{M}}$ and $\widetilde{W}$ are given by the expressions (6) and (7), respectively, after replacing $\mathbf{G}_{0}, \mathbf{G}_{0}^{\prime}$ with $\widetilde{\mathbf{G}}_{0}, \widetilde{\mathbf{G}}_{0}^{\prime}$.

Differentiating twice Eq.(14) with respect to $Q_{z}$ and performing the numerical integrations, we find $\rho_{n} / n=$ $\left(\kappa m^{2} / k_{F}\right) F\left(1 / k_{F} a\right)$, where the function $F\left(1 / k_{F} a\right)$ is plotted in Fig 2 with the solid line. We see that also the normal fluid density exhibits a nonmonotonic behavior, reaching its minimum value near unitarity, at $1 / k_{F} a \approx-0.5$.

In the BCS regime, the leading contribution to the potential (14) comes from the first term in the rhs and we find $\rho_{n}=\kappa m k_{F}^{4} / 24 \pi^{2} \Delta$, in agreement with Ref.[7]. The corresponding asymptotic behavior $F=$ $\left(e^{2} / 32\right) \exp \left(\pi / 2 k_{F}|a|\right)$ is shown in Fig 2 with the dashed line. In this limit the healing length of the gas is defined as $\xi_{B C S}=k_{F} / m \Delta$ and we obtain $\rho_{n} / n=\pi \xi_{B C S} / 8 \ell$, showing that the normal density is proportional to the healing length for $\xi_{B C S} \ll \ell$.

In the opposite BEC limit $\left(1 / k_{F} a \gg 1\right)$, the major contribution in Eq.(14) comes from the bosonic fluctuations. By expanding $\widetilde{W}, \widetilde{\mathbf{M}}$ in powers of $q$ as done before Eq.(11), we find $\rho_{n}=\kappa 4 m^{3} \Delta / 3 \pi^{2}$, which is consistent with Ref. [8]. The corresponding asymptotic behavior $F=(8 / \sqrt{3 \pi}) / \sqrt{k_{F} a}$ is plotted in Fig 2 with the dashed line.

In conclusion, we have discussed the ground state properties of a disordered superfluid Fermi gas across the
BCS-BEC crossover. We have shown that superfluidity and condensation of pairs are less affected by disorder near unitarity. Remarkably, in the BCS limit an exponentially weak disorder strongly suppresses the condensate fraction while the order parameter itself is insensitive to impurities, in agreement with Anderson's theorem.

An interesting direction for future work is to generalize the above theory at finite temperatures and to study the effect of impurities on the superfluid transition temperature $T_{c}$. For BCS superfluids, the thermodynamics of the gas (and therefore $T_{c}$ ) is insensitive to impurities, due to Anderson's theorem. In contrast, for weakly interacting Bose condensates, the transition temperature is affected by disorder [19].

We acknowledge interesting discussions with G. Falco, S. Giorgini, T. Leggett, D. Petrov, L. Pitaevskii, N. Prokof'ev, M. Randeria, G.V. Shlyapnikov and W. Zwerger. We are also grateful to A. Griffin and E. Taylor for correspondence. This work is supported by the Marie Curie Fellowship under contract EDUG-038970.

[1] S. Giorgini, L. Pitaevskii and S. Stringari, preprint condmat/0706.3360.

[2] D. Clément et al., Phys. Rev. Lett. 95, 170409 (2005).

[3] C. Fort et al., Phys. Rev. Lett. 95, 170410 (2005).

[4] T. Schulte et al., Phys. Rev. Lett. 95, 170411 (2005).

[5] U. Gavish and Y. Castin, Phys. Rev. Lett. 95, 020401 (2005).

[6] P. W. Anderson, J. Phys. Chem. Solids 11, 26 (1959).

[7] See, for instance, A.A. Abrikosov, L.P. Gorkov and I.E. Dzyaloshinski, Methods of Quantum Field Theory in Statistical Physics (Dover, New York, 1963).

[8] K. Huang and H.-F. Meng, Phys. Rev. Lett. 69, 644 (1992).

[9] S. Giorgini, L. Pitaevskii, and S. Stringari, Phys. Rev. B 49, 12938 (1994).

[10] D. E. Miller et al., Phys. Rev. Lett. 99, 070402 (2007)

[11] R. Sensarma, M. Randeria, and T.L. Ho, Phys. Rev. Lett. 96, 090403 (2006).

[12] A. Spuntarelli, P. Pieri, and G.C. Strinati, Phys. Rev. Lett. 99, 040401 (2007).

[13] P. Nozieres and S. Schmitt-Rink, J. Low. Temp Phys. 59, 195 (1985).

[14] J.R. Engelbrecht, M. Randeria, and C.A.R. Sá de Melo, Phys. Rev. B 55, 15153 (1997).

[15] E. Taylor et al., Phys. Rev. A 74, 063626 (2006); N. Fukushima et al., Phys. Rev. A 75, 033609 (2007).

[16] This result should be contrasted with the exact value $a_{M}=0.6 a$, see D.S. Petrov, C. Salomon, and G.V. Shlyapnikov, Phys. Rev. Lett. 93, 090404 (2004).

[17] L.Salasnich, N. Manini and A. Parola, Phys. Rev. A 72, 23621 (2005).

[18] G. Astrakharchik et al., Phys. Rev. Lett. 95, 230405 (2005).

[19] A. V. Lopatin and V. M. Vinokur, Phys. Rev. Lett. 88, 235503 (2002). 